\title{
Pitfall of bispectral index during intraoperative seizure -a case report-
}

\author{
Hyungdong Kim and Sae Young Kim \\ Department of Anesthesiology and Pain Medicine, School of Medicine, Keimyung University, Daegu, Korea
}

We report a case of a 42-year-old male who developed generalized tonic-clonic seizure with sudden, brief decrease in bispectral index (BIS) value while undergoing emergency kidney transplantation. Few reports have been made on intraoperative pitfall of BIS value associated with seizure. This case report suggests seizure should be taken into account as a reason for such brief fall of BIS, especially while under general anesthesia or in other specific cases in which clinical signs of seizure are unseen. (Korean J Anesthesiol 2013; 65: 449-452)

Key Words: Bispectral index, General anesthesia, Seizures.

Bispectral index (BIS) has been a useful modality to reliably assess patient's sedative-hypnotic status during anesthesia [1,2]. However, few reports have been made on abnormal BIS patterns with the patient having seizure intraoperatively $[3,4]$. We report a case of a patient with no history of epilepsy, showing abnormal patterns of BIS values with clinical seizure, while under general anesthesia for kidney transplantation.

\section{Case Report}

A 42-year-old male patient with chronic renal failure underwent emergency kidney transplantation. The patient had undergone kidney transplantation 15 years previously, but graft failure had occurred 7 years previously. Ever since then, the patient had been on hemodialysis. Preoperative laboratory findings showed serum sodium $126 \mathrm{mmol} / \mathrm{L}$ with uric acid $5.9 \mathrm{mg} / \mathrm{dl}$. The patient had no medical history of epilepsy.
Anesthesia was induced with propofol $100 \mathrm{mg}$, fentanyl 100 $\mu \mathrm{g}$, and atracurium $25 \mathrm{mg}$. After tracheal intubation, ventilation was controlled to keep end-tidal $\mathrm{CO}_{2}\left(\mathrm{ETCO}_{2}\right)$ maintained around $35 \mathrm{mmHg}$. Monitors included invasive right radial arterial blood pressure, central venous pressure through right internal jugular vein, pulse oximetry, electrocardiography, peripheral nerve stimulation applying train-of-four stimulation, body temperature using esophageal temperature probe, and bispectral index (BisVISTA, Aspect Medical Systems, Newton, MA, USA). Anesthesia was maintained with desflurane 5-6\% based on hemodynamic measurements and BIS values, $\mathrm{O}_{2}$ with Air (1: 1, $\mathrm{FiO}_{2} 60 \%$ ), and muscle relaxation was achieved by using atracurium. Fluid replacement was done with $0.45 \%$ saline.

The BIS value dropped to 40 following the induction sequences and was stably maintained 40-50 afterward. About 4 hours after the beginning of surgery, when surgery was almost over and surgeons were closing fascia, generalized tremor oc-

Received: June 14, 2012. Revised: 1st, August 6, 2012; 2nd, August 29, 2012; 3rd, September 16, 2012. Accepted: October 2, 2012.

Corresponding author: Sae Young Kim, M.D., Department of Anesthesiology and Pain Medicine, School of Medicine, Keimyung University, 194, Dongsan-dong, Jung-gu, Daegu 700-712, Korea. Tel: 82-53-250-7287, Fax: 82-53-250-7240, E-mail: mandell@dsmc.or.kr (c) This is an open-access article distributed under the terms of the Creative Commons Attribution Non-Commercial License (http:// creativecommons.org/licenses/by-nc/3.0/), which permits unrestricted non-commercial use, distribution, and reproduction in any medium, provided the original work is properly cited. 
curred along with deviation of the eyeballs to the right upper side. At that time the BIS value increased from 40 to a peak value of 62 , and then was maintained mainly in the 50s during the seizure. After the tremor, a sudden and brief fall of BIS values were noticed. The BIS value dropped from 50 to 0 within a minute, stayed at 0 for about 5 minutes, and then returned to 50 without significant hemodynamic change (Fig. 1). During these sudden changes, signal quality index stayed over 80 with no other error messages. Arterial blood gas analysis was done immediately, which showed serum sodium level of $122 \mathrm{mmol} / \mathrm{L}$. In order to correct hyponatremia, $0.45 \%$ saline was replaced with $0.9 \%$ saline. Neuromuscular blockade was not sufficiently maintained, which was below $75 \%$, since we were preparing for emergence concerning the time remaining in the surgery. When tremor occurred, an additional dose of atracurium $10 \mathrm{mg}$ was injected to stop muscle contraction. Although the generalized tremor faded, such a brief fall of BIS occurred periodically for the next 30 minutes, after which the BIS value stabilized and stayed around 40 and the patient emerged without any problem. At emergence, the patient's mental status was clear enough to allow recall of his own name.

The patient was transferred to intensive care unit (ICU) right after emergence. Fifteen minutes from emergence, the patient had a generalized tonic-clonic seizure lasting 10 minutes, followed by three more episodes, each lasting 5 minutes, every 30 minutes. The patient's laboratory findings when having seizure were uric acid $13.1 \mathrm{mg} / \mathrm{dl}$ and serum sodium $124 \mathrm{mmol} / \mathrm{L}$. The patient was immediately referred for consultation to staff neurologists. An electroencephalography (EEG) performed in postictal period showed brief and generalized 5-7 Hz theta slowing waves indicative of mild cortical dysfunction caused by metabolic disturbance, toxic state, interictal state or postictal state. Intravenous lorazepam $4 \mathrm{mg}$ was used and $\mathrm{O}_{2} 3 \mathrm{~L} / \mathrm{min}$ was supplied by

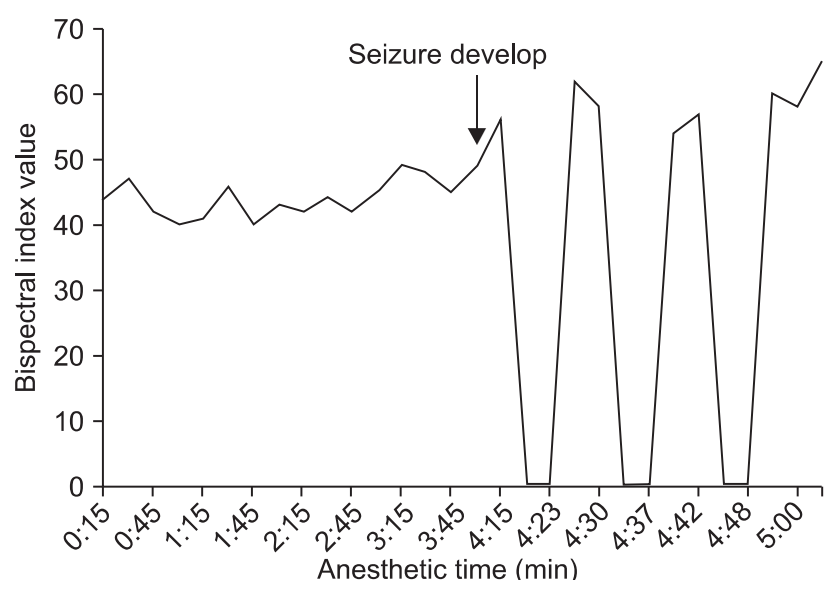

Fig. 1. Bispectral index (BIS) value during the surgery. Arrow $(\downarrow)$ is the point of seizure development. nasal cannula. The consulting neurologists diagnosed seizure by metabolic disturbance and prescribed intravenous fosphenytoin $150 \mathrm{mg}$ every 8 hours and intravenous sodium valproate $300 \mathrm{mg}$ every 12 hours. No further seizures occurred, but patient displayed a confused mental state for 10 days before normalizing. Serum sodium and uric acid normalized 2 days after surgery. Antiepileptic drugs were continued for 3 days.

\section{Discussion}

In this case, we report an abnormal intraoperative fluctuation of BIS value presenting with generalized tonic-clonic seizure.

Sudden fall of BIS value may indicate transient cerebral hypoperfusion $[5,6]$ or specific drug administration, such as neuromuscular blocking agents [7]. In this case there was no significant hypotension or hyperventilation that might have caused sudden cerebral hypoperfusion. The patient's blood pressure was maintained around 140/80 $\mathrm{mmHg}$ after an initial blood pressure of $180 / 100 \mathrm{mmHg}$. Partial pressure of $\mathrm{CO}_{2}$ of arterial blood was $37 \mathrm{mmHg}$, and there was no neck-related obstruction to cerebral blood flow. No other drugs, except for prednisolone $250 \mathrm{mg}$ administered to reduce rejection after surgery, were administered within 30 minutes from the fall in the BIS value. Other conditions that could reduce BIS value include postictal state, dementia, cerebral palsy, brain death, hypovolemia, hypoglycemia, hypothermia, and cardiac arrest [8]. The patient did not have a history of cerebral palsy or dementia. At the moment of drop in the BIS value, patient did not show any sign of dehydration. The patient received $1,500 \mathrm{ml}$ of $0.45 \%$ saline during the 4 hour period of surgery and central venous pressure was maintained at $8-10 \mathrm{mmHg}$. The heart rate was fairly constant (about 80 beats/min). Body temperature was sustained around $36.0^{\circ} \mathrm{C}$ using an air-warming blanket. With the clinical signs of generalized tonic-clonic seizure, the possibility of causes other than seizure being the reason of BIS pitfall was low.

Although BIS cannot completely substitute for a formal EEG analysis, some reports have suggested BIS as a tool to monitor the development of epileptiform EEG, patient recovery after antiepileptic treatment $[3,9]$, and also to monitor comatous patients experiencing nonconvulsive status epilepticus [10].

We were unable to obtain EEG data during this hour-long, clinically apparent seizure because the EEG was not available intraoperatively, since the operation was done the emergency setting at night. Although seizure was not firmly diagnosed with EEG of ictal state, the clinical presentation during surgery and seizure 15 minutes after emergence bore distinctive characteristics of generalized tonic-clonic seizure.

The cause of the seizure was uncertain in this case. But, hyponatremia and high serum uric acid level are two reasonable causal factors. Uremic encephalopathy is presumed to cause 
seizure by increasing neurotoxins such as guianidinosuccinic acid, methlguanidine, guanidine and creatinine in serum, cerebrospinal fluid, and brain, thus activating N-methyl-D-aspartate receptors and inhibiting gamma-aminobutyric acid(A)-ergic neurotransmission [11,12]. Hyponatremia causes neurological symptoms, such as headache, nausea, vomiting, muscle cramps, lethargy, restlessness, disorientation, and seizure, by cerebral edema and elevated intracranial pressure [13].

Presently, the patient's basal uric acid level before surgery was $5.9 \mathrm{mg} / \mathrm{dl}$ and was elevated to $13.3 \mathrm{mg} / \mathrm{dl}$ after seizure in the ICU. Reduced glomerular filtration rate, use of diuretics, and cyclosporine therapy can cause hyperuricemia after kidney transplantation [14]. In this case, blood pressure was maintained rather high considering the basal high blood pressure and intraoperative urine output after transplantation was $400 \mathrm{ml} / \mathrm{hr}$. Intravenous $15 \%$ mannitol $150 \mathrm{ml}$ was used while connecting renal artery to graft. Finally, cyclosporine was not used for immunosuppression.

Serum sodium level, on the other hand, was as low as 125 $\mathrm{mmol} / \mathrm{L}$ from the start of surgery. Right after the intraoperative seizure, the serum sodium level was $122 \mathrm{mmol} / \mathrm{L}$ and increased to $124 \mathrm{mmol} / \mathrm{L}$ during the ICU seizure. We assume that the cause of the serum sodium level decrease was a combination of $15 \%$ mannitol $150 \mathrm{ml}$ and administration of $0.45 \%$ saline 1500 $\mathrm{ml}$.

The BIS value in this case showed a unique pattern during intraoperative seizure. BIS value rose with the beginning of seizure and showed a repetitive pattern of abrupt drop to 0 and recovery to baseline. Even after atracurium injection, the cyclic pattern of BIS did not alter. We presume that the extreme changes in BIS value were caused by rapid switch of high frequency, low volt- age waves and low frequency, high voltage waves of EEG, as has been previously described [3]. Similar patterns of BIS change have been previously reported. Tallach et al. [9] reported a case of a patient whose BIS value was 93 when having seizure and 28 when controlled with propofol. Dahaba et al. [15] reported BIS change in a 14 year old patient with acute encephalitis accompanying refractory, repetitive, and partial seizure. The BIS value was $94.5 \pm 3.6$ at the start of seizures and significantly decreased to $55.1 \pm 12.5$ at the end of the seizures. In this case, BIS value was maintained at 40-50 during first 4 hours of surgery, then rapidly increased from 40 to a peak value of 62 at the beginning of seizure, then stayed in the 50s during the seizure. At the end of the seizure, the BIS value dropped to 0 where it remained for 5 minutes. Dahaba et al. [15] monitored BIS and EEG simultaneously and reported that increased BIS value with seizure is caused by epileptogenic electrical activities of brain and elevated eletromyographic activities. Rapid decreased of BIS value following seizure is explained by slow $\delta$ and $\theta$ waves dominating the post-ictal period.

Even though it is extremely rare, it is difficult to detect seizure while a patient is under general anesthesia. Intraoperative EEG is best way to detect the seizure, but is far from conventional. In this report, we suggest that BIS monitoring may be used not only to monitor sedative-hypnotic state of a patient but also to get information on seizure activity and recovery from it while under general anesthesia. Although a sudden brief fall of BIS, as in this case, does not necessarily indicate seizure activity, a repetitively reported pattern of BIS elevating during seizure and drop in the post-ictal period could be an alert that seizure is possible cause of the abnormal BIS value.

\section{References}

1. March PA, Muir WW. Bispectral analysis of the electroencephalogram: a review of its development and use in anesthesia. Vet Anaesth Analg 2005; 32: 241-55.

2. Leslie K, Davidson AJ. Awareness during anesthesia: a problem without solutions? Minerva Anestesiol 2010; 76: 624-8.

3. Chinzei M, Sawamura S, Hayashida M, Kitamura T, Tamai H, Hanaoka K. Change in bispectral index during epileptiform electrical activity under sevoflurane anesthesia in a patient with epilepsy. Anesth Analg 2004; 98: 1734-6.

4. Hamada S, Laloe PA, Hausser-Hauw C, Fischler M. Seizure after aortic clamp release: a bispectral index pitfall. J Cardiothorac Vasc Anesth 2008; 22: 119-21.

5. Morimoto Y, Monden Y, Ohtake K, Sakabe T, Hagihira S. The detection of cerebral hypoperfusion with bispectral index monitoring during general anesthesia. Anesth Analg 2005; 100: 158-61.

6. Lauwick S, English M, Hemmerling TM. An unusual case of cerebral hypoperfusion detected by bispectral index monitoring. Can J Anaesth 2007; 54: 680-1.

7. Dahaba AA, Mattweber M, Fuchs A, Zenz W, Rehak PH, List WF, et al. The effect of different stages of neuromuscular block on the bispectral index and the bispectral index-XP under remifentanil/propofol anesthesia. Anesth Analg 2004; 99: 781-7.

8. Dahaba AA. Different conditions that could result in the bispectral index indicating an incorrect hypnotic state. Anesth Analg 2005; 101: 765-73.

9. Tallach RE, Ball DR, Jefferson P. Monitoring seizures with the Bispectral index. Anaesthesia 2004; 59: 1033-4. 
10. Fernández-Torre JL, Hernández-Hernández MA. Utility of bilateral Bbispectral index (BIS) monitoring in a comatose patient with focal nonconvulsive status epilepticus. Seizure 2012; 21: 61-4.

11. Brouns R, De Deyn PP. Neurological complications in renal failure: a review. Clin Neurol Neurosurg 2004; $107:$ 1-16.

12. De Deyn PP, D'Hooge R, Van Bogaert PP, Marescau B. Endogenous guanidino compounds as uremic neurotoxins. Kidney Int Suppl 2001; 78: S77-83.

13. Adrogué HJ, Madias NE. Hyponatremia. N Engl J Med 2000; 342: 1581-9.

14. Mazali FC, Mazzali M. Uric acid and transplantation. Semin Nephrol 2011; 31: 466-71.

15. Dahaba AA, Liu DW, Metzler H. Bispectral index (BIS) monitoring of acute encephalitis with refractory, repetitive partial seizures (AERRPS). Minerva Anestesiol 2010; 76: 298-301. 